\title{
A hybrid approach for the prediction and optimization of cutting forces using grey-based fuzzy logic ${ }^{\S}$
}

\author{
Ugur Esme* \\ Mersin University/Tarsus Technology Faculty, Department of Automotive Engineering, Tarsus-Mersin
}

\begin{abstract}
This study focused on the Grey-Based Fuzzy Logic Algorithm for the prediction and optimization of multiple performance characteristics of oblique turning process. Experiments have been constructed according to Taguchi's L16 orthogonal array design matrix. Cutting speed, rate of feed and depth of cut were selected as input parameters, whereas material removal rate, cutting force and surface roughness were selected as output responses. Using grey relation analysis (GRA), grey relational coefficient (GRC) and grey relation grade (GRG) were obtained. Then, Grey based fuzzy algorithm was applied to obtain grey fuzzy reasoning grade (GFRG). Analysis of variance (ANOVA) carried out to find the significance and contribution of parameters on multiple performance characteristics. Finally, confirmation test was applied at the optimum level of GFRG to validate the results. The results also show the application feasibility of the grey based fuzzy algorithm for continuous improvement in product quality in complex manufacturing processes.
\end{abstract}

Keywords: Turning process, cutting forces, grey relation analysis, fuzzy logic algorithm, optimization.

\section{INTRODUCTION}

Turning is a very important machining process in which a single-point cutting tool removes material from the surface of a rotating cylindrical workpiece [1]. The cutting tool is fed linearly in a direction parallel to the axis of rotation [2,3]. As indicated in Figure 1, turning is carried out on a lathe that provides the power to turn the workpiece at a given rotational speed and to feed the cutting tool at a specified rate and depth of cut. Therefore, three cutting parameters, i.e. cutting speed $(V)$, feed rate $(F)$, and depth of cut $(D)$, should be properly selected for better surface finish with lower cutting force $[2,3]$.

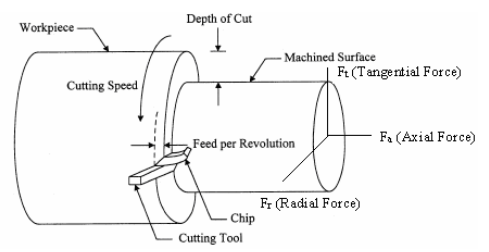

Figure 1: Schematic representation of oblique turning process [1]

In a turning operation, it is an important task to select cutting parameters for achieving high cutting performance. Usually, the desired cutting parameters are determined based on experience or by use of a handbook [1]. However, this does not ensure that the selected cutting parameters have optimal or near optimal cutting performance for a particular machine and environment. To select the cutting parameters properly, several mathematical models based on statistical regression techniques or neural computing have been constructed to establish the relationship between the cutting performance and the cutting parameters [1-8]. Then, an objective function with constraints is formulated to solve the optimal cutting parameters using optimization techniques. Therefore, considerable knowledge and experience are required for using this modern approach [1]. Furthermore, a large number of cutting experiments has to be performed and analyzed in order to build the mathematical models. Thus the required model building is very costly in terms of time and materials [1]. Basically, the grey based fuzzy logic is a powerful tool for the design of multivariable complex systems. It provides a robust systematic and efficient way in order to model the multivariable complex systems. Therefore, this study applied a Taguchi L9 orthogonal array to plan the experiments on turning process [2,3]. Three controlling factors including cutting speed $(\mathrm{V})$, depth of cut (d) and feed rate (f) were selected as input parameters whereas material removal rate, cutting force

\footnotetext{
${ }^{*}$ Corresponding authour

Email: uguresme@gmail.com (U. Eşme)

$\S$ This paper was presented in the IMSEC-2016
} 
and surface roughness were selected as output responses. Using grey relation analysis (GRA), grey relational coefficient (GRC) and grey relation grade (GRG) were obtained. Then, Grey based fuzzy algorithm was applied to obtain grey fuzzy reasoning grade (GFRG). Analysis of variance (ANOVA) carried out to find the significance and contribution of parameters on multiple performance characteristics. Finally, confirmation test was applied at the optimum level of GFRG to validate the results. The results also show the application feasibility of the grey based fuzzy algorithm for continuous improvement in product quality in complex manufacturing processes [8-12].

\section{EXPERIMENTAL PROCEDURE AND DETAILS}

The cutting experiments were carried out on an experimental lathe setup using HSS cutting tool for the machining of AISI 1050 steel bar which is $30 \mathrm{~mm}$ in diameter and $80 \mathrm{~mm}$ in length. The mechanical properties and percent composition of workpiece material is listed in Table $1[2,3]$.

Table 1. Chemical and mechanical properties of AISI 1050 medium carbon steel

\begin{tabular}{|c|c|c|c|c|c|c|c|c|}
\hline $\begin{array}{c}\text { Chemical com- } \\
\text { position \% }\end{array}$ & $\mathrm{C}$ & $\mathrm{P}$ & $\mathrm{S}$ & $\mathrm{Mn}$ & $\mathrm{Cr}$ & $\mathrm{Fe}$ & $\mathrm{Ni}$ & $\mathrm{Cu}$ \\
\hline $\begin{array}{c}\text { Mechanical pro- } \\
\text { perties }\end{array}$ & \multicolumn{2}{|c|}{ Yield strength (MPa) } & $\begin{array}{c}\text { Tensile strength } \\
(\mathrm{Mpa})\end{array}$ & $\begin{array}{c}\text { Elongation } \\
(\%)\end{array}$ & $\begin{array}{c}\text { Vickers Hardness } \\
\text { (HV) }\end{array}$ \\
\hline
\end{tabular}

Phynix TR-100 model surface roughness tester was used to measure the surface roughness of the machined samples. Cut off length () was chosen as 0.3 for each roughness measurement. Average of six measurements of surface roughness was taken to use in the multi-criteria optimization. Also, material removal rate (MRR, mm3/min) was calculated using Eq. (1);

$$
M R R=1000 V f d
$$

where $\mathrm{f}(\mathrm{mm} / \mathrm{rev})$ denotes the feed rate, $\mathrm{d}(\mathrm{mm})$ describes the cutting depth and $\mathrm{V}(\mathrm{m} / \mathrm{min})$ presents the cutting speed of the turning operation.

\subsection{Process Parameters and Test Results}

In full factorial design, the number of experimental runs exponentially increases as the number of factors as well as their level increases. This results huge experimentation cost and considerable time [11]. So, in order to compromise these two adverse factors and to search the optimal process condition through a limited number of experimental runs Taguchi's L9 orthogonal array consisting of 9 sets of data has been selected to optimize the multiple performance characteristics of turning process [8-12]. Experiments have been conducted with the process parameters given in Table 2, to obtain the machined surface on AISI 1050 medium carbon steel. The feasible space for the cutting parameters was defined by varying the cutting speed in the range of $110-600 \mathrm{~m} / \mathrm{min}$, the feed rate in the range of $0.2-0.6 \mathrm{~mm} / \mathrm{min}$, and the depth of cut in the range of $0.5-1.5 \mathrm{~mm}$.

Table 2. Cutting parameters and their levels

\begin{tabular}{|c|c|c|c|c|c|}
\hline Cutting Parameters & \multirow{2}{*}{ Notation } & \multirow{2}{*}{ Unit } & \multicolumn{3}{|c|}{ Levels of factors } \\
\cline { 4 - 6 } & & & 1 & 2 & 3 \\
\hline cutting speed & $\mathrm{V}$ & $\mathrm{m} / \mathrm{min}$ & $110^{*}$ & 300 & 600 \\
\hline feed rate & $\mathrm{f}$ & $\mathrm{mm} / \mathrm{min}$ & $0.2^{*}$ & 0.4 & 0.6 \\
\hline depth of cut & $\mathrm{d}$ & $\mathrm{mm}$ & $0.5^{*}$ & 1.0 & 1.5 \\
\hline initial factor setting
\end{tabular}

In order to prevent the sudden increase of cutting forces due to the dullness of the cutting edge, the HSS tool was changed after three repetition of each experiment. Table 3 shows the selected design matrix based on Taguchi L9 orthogonal array consisting of 9 sets of coded conditions and the experimental results for the responses of F, Ra and MRR. All these data have been utilized for analysis and evaluation of optimal parameter combination required to achieve desired quality within the experimental domain $[10,11]$. 
Table 3. Orthogonal L9 array of the experimental runs and results

\begin{tabular}{|c|c|c|c|c|c|c|}
\hline \multirow{2}{*}{ Run no } & \multicolumn{3}{|c|}{ Parameter level } & \multicolumn{3}{c|}{ Experimental results } \\
\cline { 2 - 7 } & $\mathrm{V}$ & $\mathrm{f}$ & $\mathrm{d}$ & $\begin{array}{c}\text { MRR } \\
\left(\mathrm{mm}^{3} / \mathrm{min}\right)\end{array}$ & $\begin{array}{c}\mathrm{F} \\
(\mathrm{N})\end{array}$ & $\begin{array}{c}\mathrm{R}_{\mathrm{a}} \\
(\end{array}$ \\
\hline 1 & 1 & 1 & 1 & 0.11 & 123 & 0.87 \\
\hline 2 & 1 & 2 & 2 & 0.44 & 179 & 2.33 \\
\hline 3 & 1 & 3 & 3 & 0.99 & 364 & 6.62 \\
\hline 4 & 2 & 1 & 2 & 0.60 & 166 & 1.98 \\
\hline 5 & 2 & 2 & 3 & 1.80 & 295 & 3.82 \\
\hline 6 & 2 & 3 & 1 & 0.90 & 255 & 3.96 \\
\hline 7 & 3 & 1 & 3 & 1.80 & 340 & 0.92 \\
\hline 8 & 3 & 2 & 1 & 1.20 & 218 & 1.22 \\
\hline 9 & 3 & 3 & 2 & 3.60 & 268 & 5.60 \\
\hline
\end{tabular}

\section{GREY BASED FUZZY LOGIC}

In this section, the constructed grey based fuzzy logic approach for oblique turning process optimization is given in details.

\subsection{Grey Relational Analysis (GRA)}

In Grey relational analysis, experimental data i.e., measured features of quality characteristics are first normalized ranging from zero to one. This process is known as Grey relational generation. Next, based on normalized experimental data, Grey relational coefficient is calculated to represent the correlation between the desired and actual experimental data. Then overall Grey relational grade is determined by averaging the Grey relational coefficient corresponding to selected responses [13-16]. The overall performance characteristic of the multiple response process depends on the calculated Grey relational grade (GRG) [14]. This approach converts a multiple response process optimization problem into a single response optimization situation with the objective function of overall Grey relational grade. The optimal parametric combination is then evaluated which would result highest Grey relational grade. The optimal factor setting for maximizing overall Grey relational grade can be performed by Taguchi method [13,16,17]. In Grey relational generation, the normalized F and Ra corresponding to the smaller-the-better (SB) criterion which can be expressed as given in Eq. (2) $[18,19]$ :

$$
x_{i}(k)=\frac{\max y_{i}(k)-y_{i}(k)}{\max y_{i}(k)-\min y_{i}(k)}
$$

MRR should follow the larger-the-better (LB) criterion, which can be expressed as given in Eq. (3) [18]:

$$
x_{j}(k)=\frac{y_{j}(k)-\min y_{j}(k)}{\max y_{j}(k)-\min y_{j}(k)}
$$

where $x_{i}(k)$ and $x_{j}(k)$ is the value after the Grey relational generation for SB and LB criterion respectively. Min $y_{i}(k)$ is the smallest value of $y_{i}(k)$ and for the kth response, and $\max y_{i}(k)$ is the largest value of $y_{i}(k)$ for the kth response [18]. An ideal sequence is $x_{0}(k)(\mathrm{k}=1,2, \ldots, 9)$ for the responses. The definition of Grey relational grade in the course of Grey relational analysis is to reveal the degree of relation between the 16 sequences $\left[x_{0}(k)\right.$ and $x_{i}(k), \mathrm{k}=1,2, \ldots, 9$ and $\mathrm{i}=1,2, \ldots, 9]$. The Grey relational coefficient $\xi_{i}(k)$ can be calculated as given in Eq. (4) $[13,18]$ :

$$
\xi_{i}(k)=\frac{\Delta_{\min }-\psi \Delta_{\max }}{\Delta_{0 i}(k)+\psi \Delta_{\max }}
$$

where $\Delta_{0 i}=\left\|x_{0}(k)-x_{i}(k)\right\|$ is difference of the absolute value $x_{0}(k)$ and $x_{i}(k) ; \psi$ is the distinguishing coefficient $0 \leq \psi \leq 1$ (equal $\psi=0.3$ is used); $\Delta_{\text {min }}=\forall j^{\min } \in i \forall k^{\min }\left\|x_{0}(k)-x_{j}(k)\right\|$ is the smallest value of $\Delta_{0 i}(k)$; and $\Delta_{\max }=\forall j^{\max } \in i \forall k^{\max }\left\|x_{0}(k)-x_{j}(k)\right\|$ is the largest value of $\Delta_{0 i}(k)$. After averaging the Grey relational coefficients, the Grey relational grade $\gamma_{i}$ can be computed as given in Eq. (5) [18]:

$$
\gamma_{i}=\frac{1}{n} \sum_{k=1}^{n} \xi_{i}(k)
$$

where $\mathrm{n}$ is the number of process responses. The higher value of Grey relational grade corresponds to intense relational degree between the reference sequence $x_{0}(k)$ and the given sequence $x_{i}(k)$. The reference sequence $x_{0}(k)$ represents 
the best process sequence; therefore, higher Grey relational grade means that the corresponding parameter combination is closer to the optimal [16]. The mean response for the Grey relational grade with its grand mean and the main effect plot of Grey relational grade are very important because optimal process condition can be evaluated from this plot [18].

\subsection{Fuzzy Inference System (FIS) Modeling}

In GRA, the use of performance characteristics such as lower-the-better, higher-the-better and nominal-the-better reflects that there is some level of uncertainty in the obtained results. This uncertainty can be effectively checked by using fuzzy logic [13,19-29]. The grey-fuzzy method was created and applied by Lin in 2004 [15]. It takes a fuzzy rules approach rather than making a traditional GRG estimation for grey relational analysis. For GRG estimation, two approaches were employed to compare output performance. One is the traditional GRG function while the other is the fuzzy inference system (FIS) [13,16,28-29].

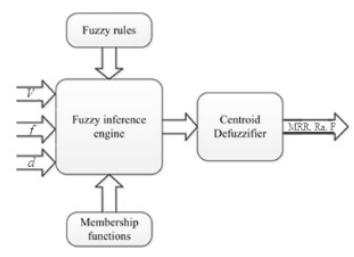

Figure 2: 3-inputs and 3-outputs fuzzy logic system

In the first step, fuzzifier uses the membership function to fuzzify inputs which are obtained before GRG calculations. Membership function is used to define how the values of the input and output are mapped to a value between 0 and 1 [14]. In the next step of the calculation, nine fuzzy rules for three inputs and one output are developed using Eq. (6) based on the results that obtained from the experiments for inference.

Rule 1 : if $\mathrm{X} 1$ is $\mathrm{A} 1$; $\mathrm{X} 2$ is $\mathrm{B} 1$; and $\mathrm{X} 3$ is $\mathrm{C} 1$ then $\mathrm{y}$ is $\mathrm{D} 1$; else

Rule 2 : if $\mathrm{X} 1$ is $\mathrm{A} 2$; $\mathrm{X} 2$ is $\mathrm{B} 2$; and $\mathrm{X} 3$ is $\mathrm{C} 2$ then $\mathrm{y}$ is $\mathrm{D} 2$; else

Rule $\mathrm{n}$ : if $\mathrm{X} 1$ is $\mathrm{An}$; $\mathrm{X} 2$ is $\mathrm{Bn}$; and $\mathrm{X} 3$ is $\mathrm{Cn}$ then y is Dn; else

$\mathrm{Ai}, \mathrm{Bi}, \mathrm{Ci}$ and $\mathrm{Di}$ are fuzzy subsets defined by the corresponding membership functions such as $\mu A_{i}, \mu B_{i}, \mu C_{i}$ and $\mu D_{i}$.

The inference engine then performs fuzzy reasoning on fuzzy rules by taking max-min inference (Eq. (7)) to generate a fuzzy value [14].

$$
\begin{aligned}
& \mu_{C_{0}}(Y)=\left(\mu_{A_{1}}\left(X_{1}\right) \wedge \mu_{B_{1}}\left(X_{2}\right) \wedge \mu_{C_{1}}\left(X_{3}\right) \wedge \mu_{D_{1}}(Y) \vee \ldots .\right. \\
& \mu_{A_{n}}\left(X_{1}\right) \wedge \mu_{B_{n}}\left(X_{2}\right) \wedge \mu_{C_{n}}\left(X_{3}\right) \wedge \mu_{D_{n}}(Y)
\end{aligned}
$$

Where $\wedge$ is the minimum operation and, $\vee$ is the maximum operation. Finally, in this study, centroid defuzzification method was used in order to convert the fuzzified values. The centroid defuzzification method is given in Eq. (8).

$$
Y_{0}=\frac{\sum Y \mu_{C_{0}}(Y)}{\sum \mu_{C_{0}}(Y)}
$$

The flowchart adopted in the present study to determine the optimal combination of ball turning parameters for the multi response optimization is shown in Figure 3.

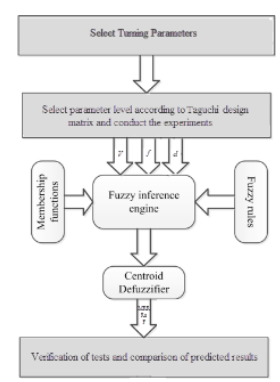

Figure 3: Flowchart of the proposed grey based fuzzy logic method 
The methodology consists of a six forward step approach given below [14]:

Step 1: Selecting the Turning input parameters and their levels as given in Table 2. Perform the experiments according to the Taguchi's orthogonal design matrix given in Table 3.

Step 2: Normalize all of the responses using Eqs. (2) and (3). Calculate the grey relational coefficients using Eq. (4), and followed by calculation of grey relational grade (GRG) using Eq. (5).

Step 3: Fuzzify the grey relational coefficients obtained from each response and the overall grey relational grade using membership function. Also, establish the fuzzy rules in linguistic form relating the grey relational coefficient and the overall grey relational grade using Eq. (6).

Step 4: Using max-min interface operations given in Eq. (7), calculate the value of fuzzy multi response output (), and follow by calculating the grey-fuzzy reasoning grade Y0 using centroid defuzzification method given in Eq. (8).

Step 5: Select the optimal combination of parameters through response table and response graph obtained from grey-fuzzy optimization. Find out the contribution of each factor and their interactions on the multi response output using analysis of variance (ANOVA) Table.

Step 6: Carry out the confirmation tests to verify the results obtained and compare the results determine the improvements and percentage error.

\section{RESULTS AND DISCUSSION}

The pre-processed data of the normalized experimental results, grey relational coefficients and the overall grey relational grade for each combination of parameters is tabulated in Table 4, Table 5 and Table 6 . The grey-fuzzy reasoning grade is obtained by using MATLAB v7.10.0 (R2010a) fuzzy logic tool box.

Table 4. Grey relational generation of each performance characteristics

\begin{tabular}{|c|c|c|c|}
\hline \multirow{2}{*}{ Run no } & MRR & F & Ra \\
\cline { 2 - 4 } & Larger-the-better & Smaller-the-better & Smaller-the-better \\
\hline Ideal sequence & 1.000 & 1.000 & 1.000 \\
\hline 1 & 0.000 & 1.000 & 1.000 \\
\hline 3 & 0.095 & 0.768 & 0.746 \\
\hline 4 & 0.252 & 0.000 & 0.000 \\
\hline 5 & 0.140 & 0.822 & 0.807 \\
\hline 7 & 0.484 & 0.286 & 0.487 \\
\hline 9 & 0.226 & 0.452 & 0.463 \\
\hline
\end{tabular}

Table 5. Evaluation of $\Delta_{0 i}$ for each of the responses

\begin{tabular}{|c|c|c|c|}
\hline Run no & MRR & F & Ra \\
\hline Ideal sequence & 1.000 & 1.000 & 1.000 \\
\hline 1 & 1.000 & 0.000 & 0.000 \\
\hline 2 & 0.905 & 0.232 & 0.254 \\
\hline 3 & 0.748 & 1.000 & 1.000 \\
\hline 4 & 0.860 & 0.178 & 0.193 \\
\hline 5 & 0.516 & 0.714 & 0.513 \\
\hline 6 & 0.774 & 0.548 & 0.537 \\
\hline 7 & 0.516 & 0.900 & 0.009 \\
\hline 8 & 0.688 & 0.394 & 0.061 \\
\hline 9 & 0.000 & 0.602 & 0.823 \\
\hline
\end{tabular}

Table 6 shows the calculated Grey relational coefficients (with the weights of $\psi_{M R R}=0.3, \psi_{F}=0.3$ and $\psi_{R}=0.3$ ) of each performance characteristic using Eq. (4). 
Table 6. Grey relational coefficient and grey relational grade of each performance characteristics

\begin{tabular}{|c|c|c|c|c|}
\hline Run no & MRR & F & Ra & Grey relational grade \\
\hline Ideal sequence & 1.000 & 1.000 & 1.000 & \\
\hline 1 & 0.248 & 1.000 & 1.000 & 0.711 \\
\hline 2 & 0.267 & 0.587 & 0.565 & 0.468 \\
\hline 3 & 0.306 & 0.248 & 0.248 & 0.264 \\
\hline 4 & 0.277 & 0.649 & 0.631 & 0.513 \\
\hline 6 & 0.390 & 0.316 & 0.391 & 0.362 \\
\hline 7 & 0.299 & 0.376 & 0.380 & 0.348 \\
\hline 8 & 0.390 & 0.268 & 0.974 & 0.538 \\
\hline 9 & 0.324 & 0.456 & 0.844 & 0.536 \\
\hline
\end{tabular}

The Grey relational coefficients, given in Table 6, for each response have been accumulated by using Eq. (4) to evaluate Grey relational grade, which is the overall representative of all the features of cutting process quality. Thus, the multi-criteria optimization problem has been transformed into a single equivalent objective function optimization problem using the combination of Taguchi approach and Grey relational analyses. Higher is the value of Grey relational grade, the corresponding factor combination is said to be close to the optimal [3,10,11].

Triangular shaped membership function, shown in Figure 4(a), is used for fuzzy modeling of the input and output data. The five linguistic membership functions such as LOWEST, LOW, MEDIUM, HIGH and HIGHEST are used to represent the GRC of the input variables.

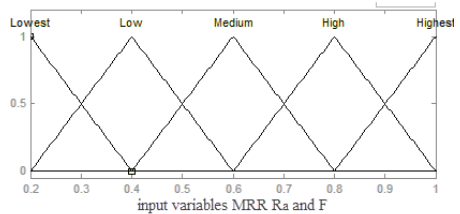

(a)

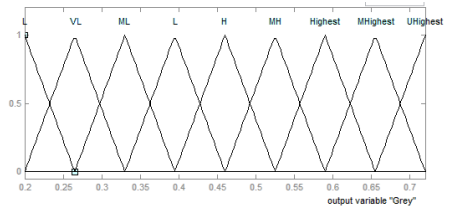

(b)

Figure 4: Constructed membership functions for (a) input parameters, (b) output parameter

GRG is represented by the nine membership functions such as LOWEST (L), VERY LOW (VL), MEDIUM LOW (ML), LOW, HIGH (H), MEDIUM HIGH (MHIGH), HIGHEST (H), MEDIUM, HIGHEST (MH) and ULTRAHIGHEST (UH). Also, the triangular shaped membership function used for GRG as shown in Figure 4(b). The values of GFRG and GRG obtained for nine experiments are shown in Table 7. It is evident that the experiment number 1 and 9 exhibit the best multiple performance characteristics with the highest GFRG. Based on the grey-fuzzy calculations, the absolute average percentage error between the GRG and GFRG was calculated as 6.05\%. Also, as shown in Figure 5 high correlation coefficient of R2=0.987 indicates the close relationship between GRG and GFRG.

Table 7. Comparison of GRG and GFRG

\begin{tabular}{|c|c|c|c|c|}
\hline Experiment no & $\begin{array}{c}\text { Grey relational grade } \\
(\text { GRG })\end{array}$ & $\begin{array}{c}\text { Grey-fuzzy } \\
\text { reasoning grade } \\
(\text { GFRG })\end{array}$ & $\begin{array}{c}\text { Ranksolute } \\
\text { Error \% }\end{array}$ \\
\hline 1 & 0.711 & 0.699 & 1 & 1.755 \\
\hline 2 & 0.468 & 0.444 & 6 & 5.440 \\
\hline 3 & 0.264 & 0.286 & 5 & 7.746 \\
\hline 4 & 0.513 & 0.460 & 7 & 11.522 \\
\hline 5 & 0.362 & 0.325 & 8 & 9.064 \\
\hline 6 & 0.348 & 0.319 & 3 & 2.476 \\
\hline 7 & 0.538 & 0.525 & 4 & 2.095 \\
\hline 8 & 0.536 & 0.525 & 2 & 3.048 \\
\hline
\end{tabular}




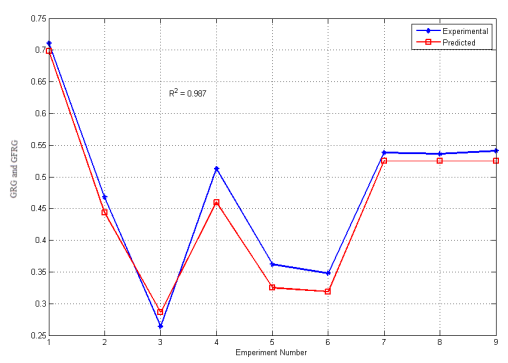

Figure 5: Comparison of experimental and fuzzy predicted GRG and GFRG

Table 8 shows the response table for the mean of GFRG. Higher is the value of GFRG, the corresponding factor combination is said to be close to the optimal [13,14]. Analysis of the means is performed for the GRFG. Based on the max-min statistics the multiple performance response is listed in Table 8. The response graph of the oblique turning parameters is plotted in Figure 8. Greater the slope of the response graph larger is the effect of the parameter on the multiple performance response [14].

Table 8. Response table for the mean Grey relational grade

\begin{tabular}{|c|c|c|c|c|}
\hline \multirow{2}{*}{ Factors } & \multicolumn{5}{|c|}{ Grey fuzzy relational grade } \\
\cline { 2 - 5 } & Level 1 & Level 2 & Level 3 & max-min \\
\hline V & 0.48 & 0.37 & 0.52 & 0.15 \\
\hline f & 0.56 & 0.43 & 0.38 & 0.18 \\
\hline d & 0.51 & 0.48 & 0.38 & 0.13 \\
\hline \multicolumn{5}{|c}{ Total mean Grey fuzzy relational grade $=0.46$} \\
\hline
\end{tabular}

As indicated in Figure 6, above the mean grey fuzzy relational grade which is shown by dashed line, the optimal condition for the turning process obtained as V3f1d1.

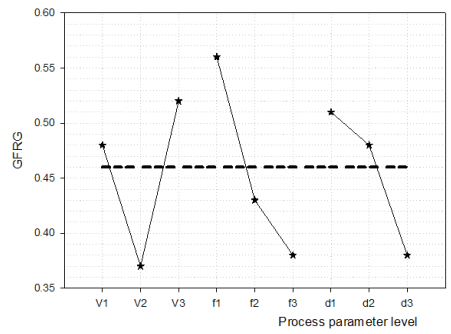

Figure 6: Response graph of GFRG for turning parameters

Analysis of variance analysis (ANOVA) is carried out to investigate which oblique turning parameters significantly affect the performance characteristic [13]. The results of ANOVA are shown in Table 9. The analysis is done at a significance level of $\alpha=0.05$ (confidence level of 95\%). Also, the statistical testing for the experimental data was carried out using Fisher's F test for ANOVA [19]. Larger the F-value shows that the change of process parameter have more strong influence on the performance characteristic [14,25]. According to the contribution effect of the parameters, feed rate $(39.13 \%)$ is found to be the major factor affecting multiple performance responses, whereas cutting speed $(28.20 \%)$ and depth of cut $(21.01 \%)$ are found to be the second and third ranking factor on the surface roughness, material removal rate and cutting force respectively. Also, the same contribution order is seen from the F test column of the Table 9.

Table 9. ANOVA results for GFRG

\begin{tabular}{|c|c|c|c|c|c|}
\hline Parameter & $\begin{array}{c}\text { Degree of } \\
\text { Freedom }\end{array}$ & $\begin{array}{c}\text { Sum of } \\
\text { Square }\end{array}$ & $\begin{array}{c}\text { Mean } \\
\text { Square }\end{array}$ & F & Contribution (\%) \\
\hline $\mathrm{V}$ & 2 & 0.039 & 0.019 & 2.39 & 28.20 \\
\hline $\mathrm{f}$ & 2 & 0.054 & 0.027 & 3.34 & 39.13 \\
\hline $\mathrm{d}$ & 2 & 0.029 & 0.015 & 1.82 & 21.01 \\
\hline Error & 2 & 0.016 & 0.008 & & 11.59 \\
\hline Total & 8 & 0.138 & & & 100 \\
\hline
\end{tabular}

After evaluating the optimal parameter settings, the next step is to predict and verify the enhancement of quality characteristics using the optimal parametric combination. The estimated grey-fuzzy reasoning grade $\gamma$ using the optimal level of the design parameters can be calculated as: given in Eq. (9) $[13,14]$. 


$$
\hat{\gamma}=\gamma_{m}+\sum_{i=1}^{o}\left(\bar{\gamma}_{i}-\gamma_{m}\right)
$$

where $\gamma_{m}$ is the total mean GFRG, $\bar{\gamma}_{i}$ is the GFRG at the optimal level, and o is the number of the main design parameters that affect the quality characteristics of turning process. Table 10 summarizes the results of confirmation test and optimum levels.

Table 10. Confirmation test results

\begin{tabular}{|c|c|c|c|}
\hline & Initial factor settings & \multicolumn{2}{|c|}{ Optimal process condition } \\
\cline { 3 - 4 } & & Prediction & Experiment \\
\hline Factor levels & V1f1d1 & V3f1d1 & V3f1d1 \\
\hline MRR (mm3/min) & 0.11 & & 0.30 \\
\hline $\mathrm{F}(\mathrm{N})$ & 123 & & 115 \\
\hline $\mathrm{Ra}(\mu \mathrm{m})$ & 0.87 & 0.68 & 0.70 \\
\hline Grey fuzzy reasoning grade $(\mathrm{GFRG})$ & 0.64 & \\
\hline \multicolumn{2}{|c|}{ Improvement in grey fuzzy reasoning grade=0.06 } \\
\hline
\end{tabular}

At the optimal setting (V3f1d1) the estimated GFRG is 0.68 and that obtained from the experiment is 0.70 which is also larger than the GFRG result of initial factor setting (0.64). Thus, a gain of 0.02 in GFRG means that the grey fuzzy logic can be successfully utilized for multi characteristics optimization problems of all the machining process $[13,14]$.

\section{CONCLUSIONS}

This study has concentrated on the grey based fuzzy logic multi response optimization in the oblique turning process. The following conclusions can be drawn from this study.

- The grey-fuzzy algorithm is suitable for optimizing the complicated multi response machining processes,

- Output turning parameters such as surface roughness, material removal rate and cutting force are greatly improved by using grey based fuzzy logic optimization,

- ANOVA analysis showed that, table feed rate has the highest contribution $(39.13 \%)$ on the multiple performance characteristics followed by the cutting speed $(28.20 \%)$ and depth of cut $(21.01 \%)$,

- The grey fuzzy optimization results of parameters for turning process of AISI 1050 medium carbon steel are summarized as $600 \mathrm{~m} / \mathrm{min}$ cutting speed, $0.2 \mathrm{~mm} / \mathrm{min}$ feed rate and $0.5 \mathrm{~mm}$ depth of cut.

\section{REFERENCES}

[1] Yang, W.H. and Tarng, Y.S. (1998). Design optimization of cutting parameters for turning operations based on the Taguchi method. Journal of Materials Processing Technology, vol. 84, no. 1, pp. 122-129. 10.1016/S0924-0136(98)00079-X

[2] Esme, U. and Serin, H. (2007). A Study for the Optimization of Cutting Forces Based on the Taguchi Method. Çukurova Üniversitesi Mühendislik Mimarlık Fakültesi Dergisi, vol. 22, no. 1, pp. 1-12.

[3] Kazancoglu, Y., Esme, U., Bayramoglu, M., Guven, O., and Ozgun, S. (2011). Multi-Objective Optimization of the Cutting Forces In Turning Operations Using the Grey-Based Taguchi Method Materiali in Tehnologije, vol. 45, no. 2, pp. 105-110.

[4] Oxley, P.L.B. (1988). Modelling machining processes with a view to their optimization and to the adaptive control of metal cutting machine tools. Robotics and Computer-Integrated Manufacturing, vol. 4, no. 1, pp. 103-119. 10.1016/07365845(88)90065-8

[5] Chryssolouris, G. and Guillot, M. (1990). A Comparison of Statistical and AI Approaches to the Selection of Process Parameters in Intelligent Machining. Journal of Engineering for Industry, vol. 112, no. 2, pp. 122-131. 10.1115/1.2899554

[6] Yao, Y. and Fang, X.D. (1992). Modelling of multivariate time series for tool wear estimation in finish-turning. International Journal of Machine Tools and Manufacture, vol. 32, no. 4, pp. 495-508. 10.1016/0890-6955(92)90041-E

[7] Zhou, C. and Wysk, R.A. (1992). An integrated system for selecting optimum cutting speeds and tool replacement times. International Journal of Machine Tools and Manufacture, vol. 32, no. 5, pp. 695-707. 10.1016/0890-6955(92)90024-B

[8] Chua, M.S., Rahman, M., Wong, Y.S., and Loh, H.T. (1993). Determination of optimal cutting conditions using design of experiments and optimization techniques. International Journal of Machine Tools and Manufacture, vol. 33, no. 2, pp. 297305. 10.1016/0890-6955(93)90081-5

[9] Disney, J. and Bendell, T. (1988). The Taguchi Approach to Designing for Reliability. in 10th Advances in Reliability Technology Symposium, G. P. Libberton, Ed. Dordrecht: Springer Netherlands, pp. 328-334. 
[10] Montgomery, D.C. (2008). Design and analysis of experiments. John Wiley \& Sons.

[11] Datta, S., Bandyopadhyay, A., and Pal, P.K. (2008). Grey-based taguchi method for optimization of bead geometry in submerged arc bead-on-plate welding. The International Journal of Advanced Manufacturing Technology, vol. 39, no. 11, pp. 1136-1143. 10.1007/s00170-007-1283-6

[12] Esme, U., Bayramoglu, M., Kazancoglu, Y., and Ozgun, S. (2009). Optimization of weld bead geometry in TIG welding process using grey relation analysis and Taguchi method. Materiali in tehnologije, vol. 43, no. 3, pp. 143-149.

[13] Esme, U., Kulekci, M.K., Ustun, D., Kahraman, F., and Kazancoglu, Y. (2015). Grey-based fuzzy algorithm for the optimization of the ball burnishing process. Materials Testing, vol. 57, no. 8, pp. 666-673. 10.3139/120.110763

[14] Pandey, R.K. and Panda, S.S. (2014). Optimization of bone drilling parameters using grey-based fuzzy algorithm. Measurement, vol. 47, pp. 386-392. 10.1016/j.measurement.2013.09.007

[15] Lin, C.L. (2004). Use of the Taguchi Method and Grey Relational Analysis to Optimize Turning Operations with Multiple Performance Characteristics. Materials and Manufacturing Processes, vol. 19, no. 2, pp. 209-220. 10.1081/AMP-120029852

[16] Yang, Y.-S. and Huang, W. (2012). A grey-fuzzy Taguchi approach for optimizing multi-objective properties of zirconium-containing diamond-like carbon coatings. Expert Systems with Applications, vol. 39, no. 1, pp. 743-750. 10.1016/j. eswa.2011.07.067

[17] Esme, U. (2010). Use of grey based Taguchi method in ball burnishing process for the optimization of surface roughness and microhardness of AA 7075 aluminum alloy. Materiali in tehnologije, vol. 44, no. 3, pp. 129-135.

[18] Hsiao, Y.F., Tarng, Y.S., and Huang, W.J. (2007). Optimization of Plasma Arc Welding Parameters by Using the Taguchi Method with the Grey Relational Analysis. Materials and Manufacturing Processes, vol. 23, no. 1, pp. 51-58. $10.1080 / 10426910701524527$

[19] Lim, S.-H., Lee, C.-M., and Chung, W.J. (2006). A study on the optimal cutting condition of a high speed feeding type laser cutting machine by using Taguchi method. International journal of precision engineering and manufacturing, vol. 7, no. 1, pp. 18-23.

[20] Kulekci, M.K., Esme, U., Ocalir, S., Ustun, D., and Kazancoglu, Y. (2016). Tensile shear strength and elongation of FSW parts predicted by Taguchi-based fuzzy logic. Materials Testing, vol. 58, no. 4, pp. 351-356. 10.3139/120.110856

[21] Zadeh, L.A. (1965). Fuzzy sets. Information and Control, vol. 8, no. 3, pp. 338-353. 10.1016/S0019-9958(65)90241-X

[22] Pattnaik, S., Karunakar, D.B., and Jha, P.K. (2013). Multi-characteristic optimization of wax patterns in the investment casting process using grey-fuzzy logic. The International Journal of Advanced Manufacturing Technology, journal article vol. 67, no. 5, pp. 1577-1587. 10.1007/s00170-012-4591-4

[23] Li, F.L., Xia, W., Zhou, Z.Y., Zhao, J., and Tang, Z.Q. (2012). Analytical prediction and experimental verification of surface roughness during the burnishing process. International Journal of Machine Tools and Manufacture, vol. 62, pp. 67-75. 10.1016/j.ijmachtools.2012.06.001

[24] Sagbas, A. (2011). Analysis and optimization of surface roughness in the ball burnishing process using response surface methodology and desirabilty function. Advances in Engineering Software, vol. 42, no. 11, pp. 992-998. 10.1016/j.advengsoft.2011.05.021

[25] Liu, N.-M., Horng, J.-T., and Chiang, K.-T. (2009). The method of grey-fuzzy logic for optimizing multi-response problems during the manufacturing process: a case study of the light guide plate printing process. The International Journal of Advanced Manufacturing Technology, journal article vol. 41, no. 1, pp. 200-210. 10.1007/s00170-008-1448-y

[26] Ahilan, C., Kumanan, S., and Sivakumaran, N. (2009). Multi-objective optimisation of CNC turning process using grey based fuzzy logic. International Journal of Machining and Machinability of Materials, vol. 5, no. 4, pp. 434-451. 10.1504/ ijmmm.2009.026902

[27] Krishnamoorthy, A., Rajendra Boopathy, S., Palanikumar, K., and Paulo Davim, J. (2012). Application of grey fuzzy logic for the optimization of drilling parameters for CFRP composites with multiple performance characteristics. Measurement, vol. 45, no. 5, pp. 1286-1296. 10.1016/j.measurement.2012.01.008

[28] Chiang, K.-T. and Chang, F.-P. (2006). Application of grey-fuzzy logic on the optimal process design of an injection-molded part with a thin shell feature. International Communications in Heat and Mass Transfer, vol. 33, no. 1, pp. 94-101. 10.1016/j. icheatmasstransfer.2005.08.006

[29] Jean, M.-D., Lin, B.-T., and Chou, J.-H. (2006). Design of a fuzzy logic approach for optimization reinforced zirconia depositions using plasma sprayings. Surface and Coatings Technology, vol. 201, no. 6, pp. 3129-3138. 10.1016/j.surfcoat.2006.06.056 